\title{
Self-organized evolution in a socioeconomic environment
}

\author{
A. Arenas, ${ }^{1}$ A. Díaz-Guilera, ${ }^{2}$ C. J. Pérez, ${ }^{2}$ and F. Vega-Redondo ${ }^{3}$ \\ ${ }^{1}$ Departament d'Enginyeria Informàtica, Universitat Rovira i Virgili, Carretera Salou s/n, E-43006 Tarragona, Spain \\ ${ }^{2}$ Departament de Física Fonamental, Universitat de Barcelona, Diagonal 647, E-08028 Barcelona, Spain \\ ${ }^{3}$ Facultad de Económicas and Instituto Valenciano de Investigaciones Económicas, Universidad de Alicante, E-03071 Alicante, Spain
}

(Received 26 May 1999)

\begin{abstract}
We propose a general scenario to analyze technological changes in socio-economic environments. We illustrate the ideas with a model that incorporating the main trends is simple enough to extract analytical results and, at the same time, sufficiently complex to display a rich dynamic behavior. Our study shows that there exists a macroscopic observable that is maximized in a regime where the system is critical, in the sense that the distribution of events follow power laws. Computer simulations show that, in addition, the system always self-organizes to achieve the optimal performance in the stationary state.

PACS number(s): 87.23.Ge, 02.50.Le, 05.65.+b, 87.23.Kg
\end{abstract}

The evolution of socioeconomic environments is attracting the interest of the physics community due to the inherent complexity of many dynamic processes. In particular, concepts and tools widely used in nonequilibrium statistical physics have proved to be quite useful when studying the complex behavior of interacting economic agents [1-3].

There are clear evidences that social and economic change in modern societies typically come in "waves" with seemingly little intertemporal structure. There are many factors that can contribute to such complex evolution but, in essence, any theory able to account for the inherent dynamics of the phenomenon should consider how the stimulus for change spreads by gradual local interaction through a social network as well as the incentives that govern individual behavior [4-7]. The hope is that, independently of the particular choice for the microscopic rules describing the dynamic behavior of the agents that form an arbitrary system, one should observe some collective trends that could be reflected in terms of macroscopic observables.

In this paper, our main goal is to define a general scenario that could be useful to understand evolution in socioeconomic environments and within such a broad field our concern is related to technological progress. In a general sense, let us consider a population of agents each of them interacting with a group of neighbors in order to carry out projects of mutual interest. From these collaborations agents obtain payoffs which, of course, tend individually to be as large as possible. To be more precise these payoffs should reflect several basic properties. First, they should account for a basic benefit obtained just for having a certain technological level. It might be thought as an index for the technological potential productivity. It is reasonable to assume that the higher the technological level the larger the base payoff will be. Furthermore, it should measure how similar the tools required to undertake a mutual project are. It should favor those collaborations where both technological levels are very similar (high compatibility) and punish any waste of resources derived from a possible mismatch between them. In other words, technological compatibility should induce high values of the payoff function while significant costs should arise from any degree of incompatibility [8]. It is also reasonable to assume that those costs are bounded from below (the bankrupt).
The dynamics must be consequent with the aforementioned basic trends. Two main ingredients contribute to the dynamical evolution. One is the interaction with the rest of the population. Each agent should have the possibility to modify her technological level if the benefits derived from this change are increased. With only this term the system might reach a quiescent state where all the agents are happy with their respective technological level, not necessarily the same for all of them. To complete the picture, it is also natural to think of individual mechanisms of technological improvement which could be modeled as a sudden update of the state of a given agent. This change plays the role of a perturbation and admits several interpretations (e.g., local innovation, a shock in payoffs, population renewal, etc.). Immediately, her nearest neighbors check whether an update to a new technological state is more profitable for them. The process can be extended all over the network triggering a wave of change or avalanche till a new quiescent state is reached. Then, the sequence of events is repeated again. Notice that in modern socioeconomic environments, the diffusion of information and technology is usually a fast process while advances are developed in a much slower time scale. Therefore, it is reasonable to assume that both processes are defined in different time scales. Other ingredients can also be incorporated into this general framework but, up to now, let us keep this simple picture in mind.

Next point concerns the characterization of progress. More precisely, we need to measure things in terms of a macroscopic observable. Accounting for the rate of advance, the most natural choice is the mean velocity of progress, which can be defined as

$$
\rho=\lim _{T \rightarrow \infty} \rho(T)=\lim _{T \rightarrow \infty} \frac{\sum_{t=1}^{T} H(t)}{\sum_{t=1}^{T} s(t)},
$$

where $s(t)$ is the number of agents involved in the avalanche generated at time $t$, and $H(t)$ is the total advance induced by the wave on the whole population. Assuming that each individual update induces a certain cost, $\rho$ also measures the 
total cost needed to reach a certain global (averaged) technological level. For instance, a high advance rate will imply to achieve some given level at minimum total cost, i.e., with the minimal number of individual updates.

Several questions arise in a natural manner. For a given system defined in such scenario, is there any kind of behavior which could optimize $\rho$ ? If this is the case, how does the system evolve towards the optimal state?

Let us steer the intuition of the reader with a physical discussion on the basis of the scenario proposed so far. If the cost of an update is very small, then all agents are willing to adopt the best available technology and any new particular improvement will immediately be diffused through the whole population, leading to an avalanche involving a large number of individuals, eventually the whole system. In contrast, in the opposite case it is generally difficult to find an agent interested in changing her current state since even if a rather advanced technology is available, the cost will typically be too high leading to a situation where avalanches tend to be very small. These two extreme situations can be identified with a supercritical or subcritical regime, respectively. In both cases the advance rate defined in Eq. (1) tends to be independent of the number of agents $n$. From a theoretical standpoint one might expect that in the intermediate range the most interesting phenomena can emerge since it is where rich dynamic behavior may flourish. In this regime, we expect that a substantial degree of heterogeneity (but one that can be eventually broken by the advance of technological avalanches) plays a fundamental role.

Once one knows the distribution of $s$ and $H$ then it is straightforward to work out $\rho$ and determine if there is any specific regime where the performance of a given system is optimal. There are plenty of evidences reported in the literature showing that quantities such as $s$ and $H$ follow power law distributions [1,9-15]. Therefore, we assume that the avalanche-size distribution obeys a power law $P(s) \sim 1 / s^{\gamma}$, for some $\gamma>0$, as well as the distribution of technological advances per avalanche $P(H) \sim 1 / H^{\beta}$ for some positive $\beta$. In addition, we take the natural assumption of considering that avalanche sizes and induced advances should also be related, on average, through some power relationship of the form

$$
H \sim s^{\alpha}
$$

with $\alpha \geqslant 1$. Notice that the lower bond $\alpha=1$ corresponds to the two aforementioned extreme situations: either a uniform growing front (supercritical) or hardly interacting agents (subcritical). It can be easily shown that, provided $\gamma$ and $\beta$ are larger than 1 , the following relation should hold among the exponents [10]:

$$
\alpha=\frac{\gamma-1}{\beta-1} .
$$

It is also straightforward to find that the rate of technological progress is

$$
\rho=\frac{2-\gamma}{\alpha-\gamma+1} \frac{n^{1+\alpha-\gamma}-1}{n^{2-\gamma}-1},
$$

where $n$ is the size of the system (number of agents). For large $n$, three different regimes can be considered.

(a) $\gamma<2$ :

$$
\rho=\frac{2-\gamma}{\alpha-\gamma+1} n^{\alpha-1},
$$

(b) $2<\gamma<\alpha+1$ :

$$
\rho=\frac{\gamma-2}{\alpha-\gamma+1} n^{\alpha+1-\gamma},
$$

(c) $\gamma>\alpha+1$ :

$$
\rho=\frac{\gamma-2}{\gamma-\alpha-1} .
$$

Several conclusions can be extracted from these expressions. First, notice that there are two regimes where the rate of technological advance increases with the size of the system which suggests that large ensembles enjoy beneficial "scale effects," i.e., large populations grow faster. It is in these regions where one could expect an optimal advance rate and therefore an optimal collective performance. In contrast, there is another regime where $\rho$ is independent of $n$ suggesting a poorer cooperative dynamics.

Let us illustrate the general scenario by proposing a particular model endowed with the essential features requested in the introduction. In this way we can investigate how the distribution of technological advances depends on microscopic details of the dynamics. In order to enhance transparency we have reduced the complexity of the model as much as possible. We have considered a system formed by a population of $n$ agents defined on a periodic 1D geometry and nearest neighbor interactions. Each agent is characterized by a positive real variable $a_{i}(t)$ identified as the technological level. The interaction with the neighbors is evaluated in terms of the payoff function that we have chosen to be

$$
\psi\left(a, a^{\prime}\right)= \begin{cases}a-k_{1}\left(1-e^{-\left(a-a^{\prime}\right)}\right) & \text { if } a>a^{\prime} \\ a-k_{2}\left(1-e^{-\left(a^{\prime}-a\right)}\right) & \text { if } a<a^{\prime} .\end{cases}
$$

Thus, the base payoff obtained from using a certain technology is assumed equal to $a$ while the incompatibility costs resulting from being too advanced or too backwards relative to neighbors are parametrized, respectively, by positive factors $k_{1}$ and $k_{2}$. However, as we will see later, the overall properties of the system only depend on the difference $k$ $=k_{1}-k_{2}$.

The dynamics of the model has two main components. At each time step a randomly selected agent is chosen to update her technological level from $a_{i}(t-1)$ to $a_{i}(t)=a_{i}(t-1)$ $+\tilde{\sigma}_{i}(t)$ where $\tilde{\sigma}_{i}(t)$ is a i.i.d. random variable. The $j=i$ \pm 1 agent now has three options: either to maintain her level or to adopt the level of one of her two neighbors. She is assumed to take that action $a=\left\{a_{j}, a_{j-1}, a_{j+1}\right\}$, which maximizes her total payoff $\psi\left(a, a_{j-1}\right)+\psi\left(a, a_{j+1}\right)$ [16]. This process continues until no agent wants to perform any adjustment in her technological level. At each time step, the size of the avalanche is 


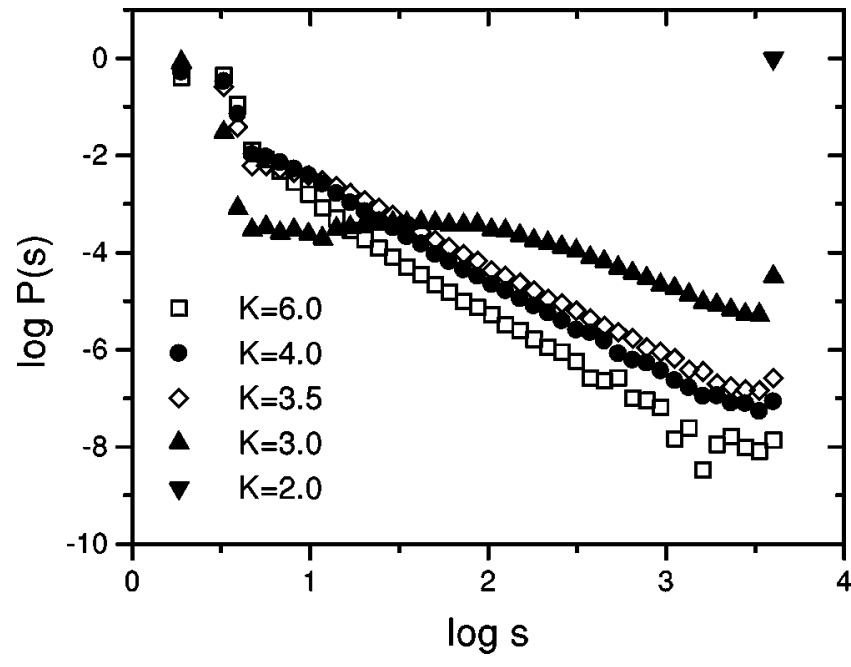

FIG. 1. Logarithm of the probability of having an avalanche of size $s$ vs. logarithm of the size $s$, for different values of $k=k_{1}$ $-k_{2}$. The length of the system is kept fixed at $n=4096$. For $k=2$ only events of size $n$ are observed.

$$
s(t) \equiv \#\left\{i: a_{i}(t) \neq a_{i}(t-1)\right\},
$$

where $\#\{\}$ stands for the cardinality of the set in question, and the total advance

$$
H(t) \equiv \sum_{i=1}^{n}\left[a_{i}(t)-a_{i}(t-1)\right]
$$

Then, again one agent is updated randomly and so on.

Three different regimes are clearly observed in simulations. Figure 1 shows the distribution of avalanches for different values of the parameter $k$. As we expected, below a certain critical value all the avalanches are of the size of the system. The technology advances at unison similar to a uniform front. Any perturbation in the system due to innovations is incorporated immediately by the rest of agents because the cost of the update is very small. In the opposite situation, i.e., for large $k$, the avalanches are of small size since the agents behave almost independently; this behavior is close to the random deposition model well known in surface growth [17]. For intermediate values of the coupling parameter the distribution of avalanches follows a power law for several orders of magnitude of avalanche sizes. The technological advance is also distributed according to a powerlaw and assumption (2) is also fulfilled as we show in Fig. 2. Therefore, in this regime there is a clear absence of time as well as length scales typical of a critical state $[9,10]$. We have thus shown that different regimes $(\mathrm{A}),(\mathrm{B}),(\mathrm{C})$ appear in this very simple model when changing $k$.

A deeper analysis of the model shows that it is possible to extract analytical information about the location of the supercritical regime. In particular, by only using local arguments, it is straightforward to show that if the difference in technological level between two neighboring sites of a given agent $i$, denoted by $\Delta=\left\|a_{i+1}-a_{i-1}\right\|$, satisfies

$$
k \equiv k_{1}-k_{2}<k^{*}(\Delta) \equiv \frac{2 \Delta}{1-e^{-\Delta}},
$$

then agent $i$ will always choose the highest local technological level which in its turn can trigger additional updates in neighboring sites [16]. In our case, where perturbations are assumed uniformly random distributed in the interval $[0,1]$ and consequently the difference between agents is a continuous variable, when $k \leqslant k^{*}(0)=2$ any local inhomogenenity cannot be sustained and the system achieves a global synchronized state where the technological level of all the agents is exactly the same.

Now, let us consider the evolution of the macroscopic observable $\rho$. Figure 3 shows the time evolution of $\rho$ for different values of $k$ and a fixed system size $n=512$, whereas Fig. 4 displays the stationary (long run) values of $\rho$ for different values of $k$ and different system sizes. Two important and appealing features must be singled out here. First, Fig. 3 shows that $\rho$ grows monotonically over time, the system

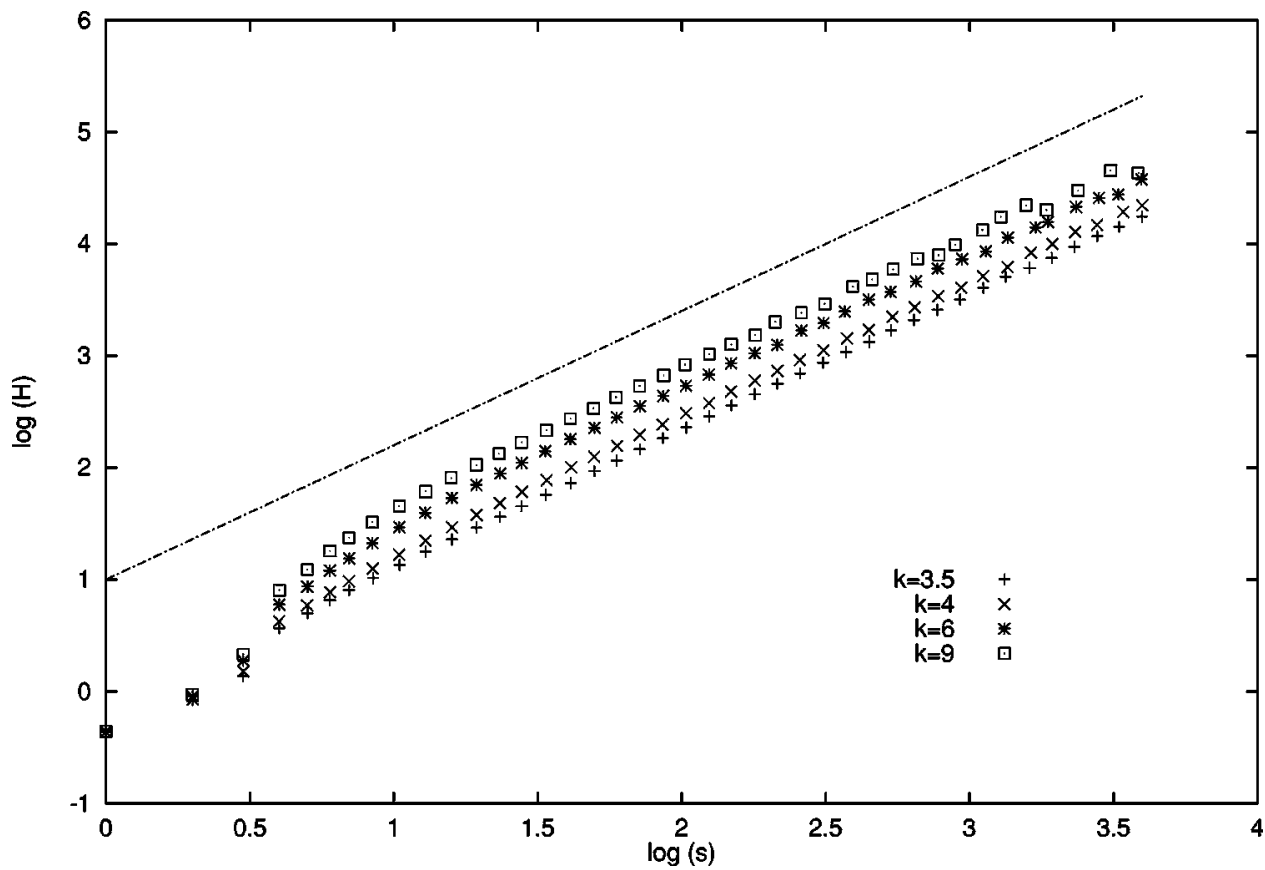

FIG. 2. Relationship $s-H$ for different values of $k$. The system size and the number of updates are the same as those of Fig. 1. The straight line has a slope 1.2 . 


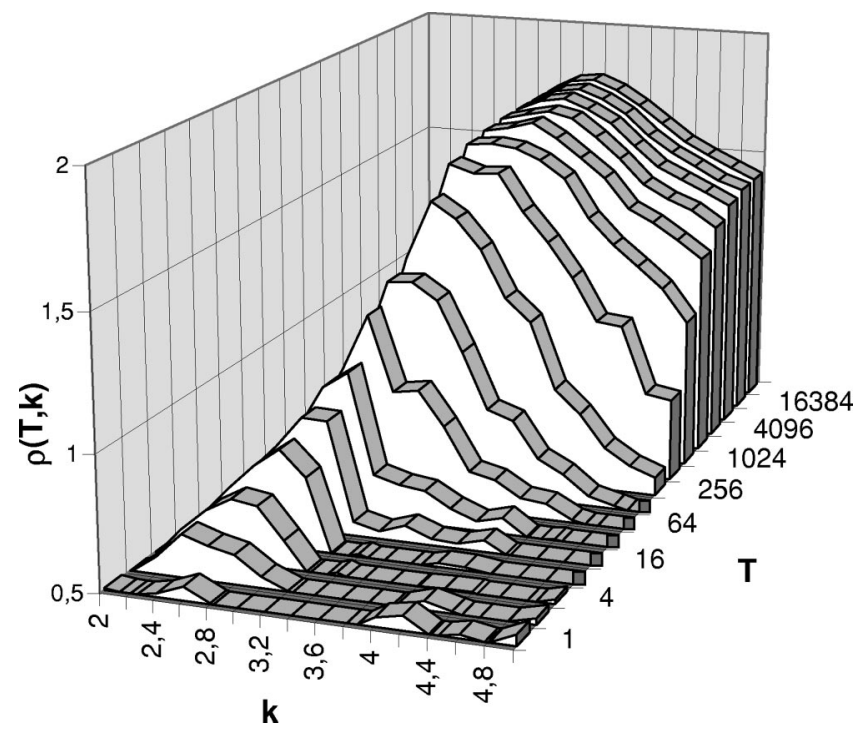

FIG. 3. Time evolution of $\rho$ as a function of $k$. The number of updates, $T$, accounts for the slow time scale. The system size is kept fixed at $n=512$. The result is an average over 100 independent runs.

self-organizes to achieve, for each $k$ the best associated performance. No matter what is the initial condition the system evolves to maximize the advance rate. This quantity is maximal when the stationary state is reached. As far as we know, this is the first model where critical behavior is attained through a process of self-organization that maximizes a certain macroscopic observable. Second, Fig. 4 indicates that $\rho$ is maximized within the critical region, at a point located on its "lower edge" [18] (i.e., within the narrow range where $k \in[3,4])$. Notice that in the two limit cases $k \leqslant 2$ and $k$ $\rightarrow \infty, H \approx s$ and therefore $\rho$ is equal to the expected value of the external random perturbation, 0.5 in our case. Furthermore, these figures clearly show that the advance rate depends positively on the number of agents $n$ stressing again the faster growth of large economies. This fact is the motivation to investigate also if the model presented in this work accomplishes the relationship between exponents predicted in the general scenario. We have indeed confirmed that the scaling relation (3) is fulfilled within numerical accuracy, by

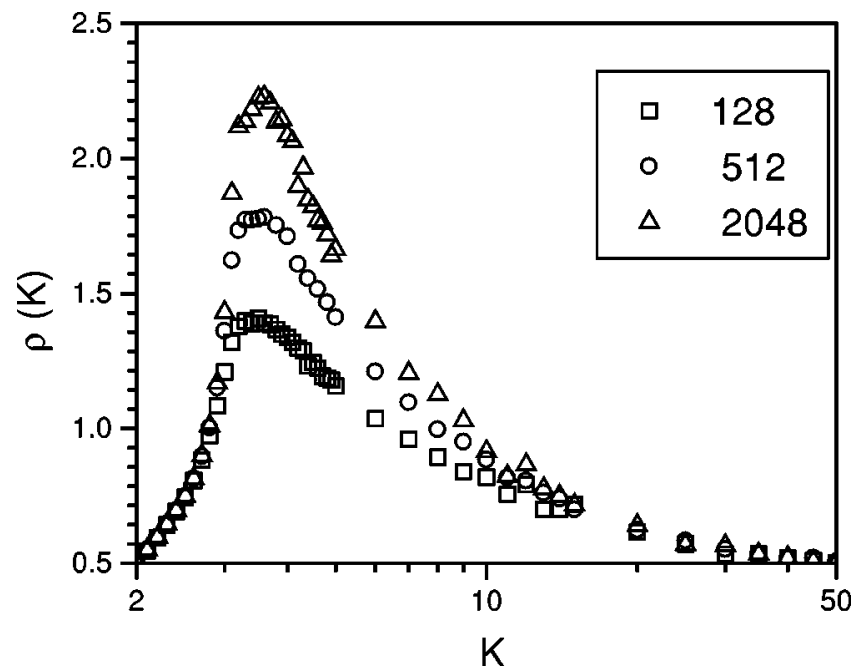

FIG. 4. Advance rate as a function of $k$, in a log-log scale, for three different values of the length. For each run we have generated $64 * n$ avalanches and averaged over 10 independent realizations of the noise, except for $n=2048$ where only two independent realizations have been considered.

fitting straight lines to the wide region where power-laws are observed [16].

In conclusion, we have presented here a general scenario for the study of the technological evolution in a socioeconomic environment. It is quite appealing to realize that in a very general manner the framework described in this paper is able to predict the existence of different regimes depending on the cost associated to the improvement or diffussion of technology and that these regimes can be computed directly from a macroscopic quantity without specifying details about the underlying microscopic dynamics and payoff functions. Even more, we have shown through a simple model that critical behavior is attained in a natural way through a process of self-organization that maximizes a macroscopic observable: the advance rate.

The authors gratefully acknowledge A. Mas-Colell and H.J. Jensen for helpful comments. This work was supported by DGES of the Spanish Government, Grant Nos. PB960168 and PB97-0131, and EU TMR Grant No. ERBFMRXCT980183.
[1] The Economy as an Evolving Complex System II, edited by W.B. Arthur, S.N. Durlauf, and D.A. Lane (Addison-Wesley, Reading, MA, 1997).

[2] J.P. Bouchaud and M. Potters, Theorie des Risques Financiers (Eyrolles, Alea-Saclay, 1997).

[3] Since this is a fast growing field we should point the reader to the Internet pages devoted to the Econophysics literature http:// www.unifr.ch/econophysics/, where an exhaustive list of references is permanently updated.

[4] L.E. Blume, Games Econ. Behavior 5, 387 (1993).

[5] G. Ellison, Econometrica 4, 1047 (1993).

[6] M. Kandori, G. Mailath, and R. Rob, Econometrica 61, 29 (1993).

[7] P. Young, Econometrica 61, 57 (1993).

[8] J. Farrel and J. Saloner, Rand J. Econ. 16, 70 (1985).

[9] P. Bak, How Nature Works: The Science of Self-Organized
Criticality (Copernicus, New York, 1996).

[10] H.J. Jensen, Self-Organized Criticality (Cambridge University Press, Cambridge, 1998).

[11] P. Krugman, The Self-organizing Economy (Blackwells, Cambridge, MA, 1996).

[12] R. Axtell (unpublished).

[13] S. Ispolatov, P.L. Krapivsky, and S. Redner, Eur. Phys. J. B 2, 267 (1998), and references therein.

[14] S. Redner, Eur. Phys. J. B 4, 131 (1998).

[15] D. Sornette and D. Zajdenweber, Eur. Phys. J. B 8, 653 (1999).

[16] A. Arenas, A. Díaz-Guilera, C.J. Pérez, and F. Vega-Redondo (unpublished).

[17] A.-L. Barabasi and H.E. Stanley, Fractal Concepts in Surface Growth (Cambridge University Press, Cambridge, 1995).

[18] S. Kaufman, The Origins of Order: Self Organization and Selection in Evolution (Oxford University Press, Oxford, 1993). 